\title{
SPR Biosensing MUA/Poly-L-lysine Platform for the Detection of 2,4-Dinitrophenol as Small Molecule Model System
}

\author{
M. Antonieta Daza Millone, ${ }^{1}$ Eduardo A. Ramirez, ${ }^{1}$ Cecilia Y. Chain, ${ }^{1}$ Andrea Crivaro, ${ }^{2}$ \\ David Romanin, ${ }^{2}$ Martín Rumbo, ${ }^{2}$ Guillermo Docena, ${ }^{2}$ Mauro D. Cocco, ${ }^{3}$ María L. Pedano, ${ }^{3}$ \\ Alejandro Fainstein, ${ }^{3}$ Jorgelina Montoya, ${ }^{4}$ María E. Vela, ${ }^{1}$ and R. C. Salvarezza ${ }^{1}$ \\ ${ }^{1}$ Instituto de Investigaciones Fisicoquímicas Teóricas y Aplicadas (CONICET-UNLP), CC16 Suc4, No. 1900, La Plata, \\ Buenos Aires, Argentina \\ ${ }^{2}$ Instituto de Estudios Inmunológicos y Fisiopatológicos (CONICET-UNLP), La Plata, Argentina \\ ${ }^{3}$ Centro Atómico Bariloche, Instituto Balseiro, CNEA, 8400 S. C. de Bariloche, Rio Negro, Argentina \\ ${ }^{4}$ EEA Anguil (INTA), Ruta Nac. No. $5 \mathrm{~km}$ 580, CC 11 (6326), Anguil, La Pampa, Argentina
}

Correspondence should be addressed to Cecilia Y. Chain; yamil.chain@gmail.com

Received 24 June 2016; Revised 4 October 2016; Accepted 19 October 2016

Academic Editor: Felix J. Seo

Copyright (c) 2016 M. Antonieta Daza Millone et al. This is an open access article distributed under the Creative Commons Attribution License, which permits unrestricted use, distribution, and reproduction in any medium, provided the original work is properly cited.

\begin{abstract}
Surface Plasmon Resonance assays are being developed as alternative biodetection methods for a great number of pesticides and toxins. These substances typically have low molecular weight, making it necessary to perform competitive inhibition immunoassays. In most of the cases, the strategy is to immobilize a protein derivative of the analyte, which usually involves the appearance of nonspecific protein binding which limits the detection range of the assay. In this work we present results of a poly-L-lysine (Au-MUA-PLL) based sensor platform for quantitative determination of 2,4-dinitrophenol as model system for small molecular weight substances detection. The prepared sensor chip was characterized by means of Atomic Force Microscopy, Surface Plasmon Resonance, and Surface Enhanced Raman Spectroscopy. Experiments verified the absence of nonspecific protein adsorption to Au-MUA-PLL surfaces and the improvement of the competitive inhibition assays performance in comparison with single and mixed thiol self-assembled monolayers. The possibility of directly immobilizing 2,4-dinitrophenol to the poly-L-lysine containing platforms leads to an improvement in the detection of the soluble analyte by the competitive inhibition assay avoiding undesirable nonspecific protein adsorption. Therefore, Au-MUA-PLL surfaces constitute a suitable alternative for quantitative detection of small molecules when nonspecific adsorption cannot be avoided.
\end{abstract}

\section{Introduction}

Biosensors based in Surface Plasmon Resonance (SPR) have become increasingly employed in analytical determinations of a broad range of biological molecules, pesticides, environmental pollutants, explosives, pharmacological compounds, and substances employed in medical diagnosis [1-7]. SPR biosensing is a rapid label-free method and provides the specificity and high sensitivity required for ultrasensitive analytical determinations [8-12].

The most notable attraction in SPR-based immunosensors is the highly specific detection of small molecules with extraordinarily low detection limits for a wide variety of analytes in complex matrices [7, 13-18]

Most of the reported SPR developments correspond to competitive inhibition immunoassays. A usual strategy comprises the immobilization of a protein-analyte derivative (the carrier protein is typically serum albumin) and the evaluation of the binding of anti-analyte antibodies in a mixed solution containing the analyte in unknown concentration. In order to expand the analyte concentration working range, it is of utmost importance to minimize nonspecific antibody binding, either to the sensor platform or to the analyte derivative. For this purpose, several polymer coated 
sensor chips have been prepared for optimization of signal enhancement and minimization of fouling during sensing of biological species $[19,20]$.

The reported polymers, which require three or four days to complete their preparation, showed a diminution of nonspecific protein binding when compared to the widespread carboxymethyl dextran substrates. However, a considerable amount of this undesirable effect remains in every case, probably due to the tridimensional conformation of the polymers (allowing physical trap of proteins) and the presence of negative charges that interact electrostatically with proteins that typically have positive charges at the usual working $\mathrm{pH}$.

With the aim of minimizing the nonspecific protein binding, the coating of the sensor chips should reduce both the electrostatic and the steric interactions with proteins. In this sense, poly-L-lysine (PLL) appears as interesting coating to investigate as it is a polycation at usual working $\mathrm{pHs}$. This homopolypeptide has been covalently attached via amide bonds to an alkanethiol self-assembled monolayer (SAM) on a gold surface [21], resulting in layers with typical thickness that are in accordance with the formation of a PLL monolayer [21]. The attached PLL resists desorption under extreme conditions of $\mathrm{pH}$ and ionic strength or when a large number of the PLL amino groups are derivatized with a neutral or negatively charged moiety [21], making the Au-MUAPLL platforms suitable for SPR experiments. PLL can be modified either by reaction of the amine groups with various molecules or by the replacement of some lysine residues with other amino acids so that it may be coupled to antigens, immunoglobulins, receptors, or nucleic acids to form the functional basis of a variety of biosensors. Furthermore, PLL has been reported to obtain SPR sensor platforms suitable to trap bacteria [22].

In the present study, the Au-MUA-PLL sensor performance for the detection of 2,4-dinitrophenol (DNP) as a model system for small molecule was explored [23-26]. The prepared sensor chip is characterized by means of Atomic Force Microscopy, Surface Plasmon Resonance, and Surface Enhanced Raman Spectroscopy. This work investigates the ability of the aforementioned layer to decrease the nonspecific antibody binding in comparison with surfaces covered with single 11-mercaptoundecanoic acid (MUA) and mixed thiol monolayers formed by MUA and DL-dithiothreitol (DTT). MUA-PLL coating proved to have negligible amount of nonspecific binding. The improvement of competitive inhibition immunoassays performed of SPR sensor chips coated with MUA-PLL is also demonstrated.

\section{Experimental}

2.1. Reagents and Materials. Bovine Serum Albumin (BSA), 11-mercaptoundecanoic acid (MUA), DL-dithiothreitol (DTT), poly-L-lysine (PLL) solution $0,1 \%(\mathrm{w} / \mathrm{v})$ in water of molecular weight $150-300 \mathrm{kDa}, \mathrm{N}$-hydroxysuccinimide (NHS), ethanolamine hydrochloride, N-(3-dimethylaminopropyl)- $\mathrm{N}^{\prime}$-ethylcarbodiimide hydrochloride (EDC), sodium 2,4-dinitrobenzenesulfonate (DNBS), and Tween 20 were purchased from Sigma-Aldrich. Potassium chloride, sodium chloride, sodium dihydrogen phosphate, and disodium hydrogen phosphate were from J.T. BAKER. Sodium carbonate was from Biopack.

Commercial gold substrates (SPR102-AU) were obtained from Bionavis ${ }^{\mathrm{TM}}$.

Monoclonal IgG1 anti-DNP 112D5 [27], from here referred to as "anti-DNP," was obtained following the technique described by Galfrè and Milstein [28]. Briefly, the antiDNP monoclonal antibody was obtained by fusing spleen cells from BALB/c mice immunized with HGG-DNP and Freund adjuvant, with mouse NSO myeloma cells.

BSA-DNP conjugate was synthetized following a procedure adapted from the literature [29]. Briefly, $20 \mathrm{mg}$ of BSA in $1.1 \mathrm{~mL}$ of $\mathrm{NaCl} 0.9 \%$ was mixed with $20 \mathrm{mg}$ of DNBS in $1 \mathrm{~mL}$ of Milli-Q ${ }^{\circledR}$ water. After the addition of $20.7 \mathrm{mg}$ of sodium carbonate to the resulting solution, the conjugation mixture was stirred at room temperature for 24 hours and then dialyzed against $10 \mathrm{mM}$ Phosphate Saline Buffer (PBS) $\mathrm{pH} 7.4$, before storage at $4^{\circ} \mathrm{C}$. UV-visible spectral data and MALDI-TOF were used to confirm the structures of the final conjugates (Figures S1 and S2, Supplementary Information available online at http://dx.doi.org/10.1155/2016/5432656).

2.2. Preparation of the Sensor Surfaces. Sensor surfaces covered with PLL were obtained from single MUA selfassembled monolayers as described in [21]. Briefly, MUA SAMs were exposed to $200 \mu \mathrm{L}$ of an aqueous solution of $0.1 \mathrm{mM}$ EDC and $0.04 \mathrm{mM}$ NHS for 20 minutes at $4^{\circ} \mathrm{C}$. After rinsing it with Milli-Q water, the surface was exposed to $200 \mu \mathrm{L}$ of poly-L-lysine $1 \mathrm{mg} / \mathrm{mL}$ aqueous solution ( $\mathrm{pH} 8$ ) for 90 minutes at room temperature $\left(21^{\circ} \mathrm{C}\right)$.

Single thiol SAMs were obtained by overnight incubation of the gold substrates in $50 \mu \mathrm{M}$ MUA ethanolic solution at room temperature. Mixed thiol SAMs were prepared by incubation of the gold substrates in $(1: 20) 50 \mu \mathrm{M}$ MUA : $100 \mu \mathrm{M}$ DTT ethanolic solutions during 30 minutes. In every case, the obtained surfaces were washed with Milli-Q water and dried in a stream of nitrogen before SPR measurements.

\subsection{Immobilization Procedures. The immobilization of DNP} to Au-MUA-PLL was achieved by exposing the surface to DNBS $1 \mathrm{mg} / \mathrm{mL} \mathrm{pH} \mathrm{10,} \mathrm{for} 20$ hours at room temperature $\left(21^{\circ} \mathrm{C}\right)$ outside the SPR device, a procedure based on a previous report [30]. Light exposure was prevented during conjugation. The resulting Au-MUA-PLL-DNP platform was rinsed with Milli-Q water before placing it into the SPR chipholder.

The immobilization of 2,4-dinitrophenol-bovine serum albumin conjugates (BSA-DNP) to single and mixed SAMs was performed by the well-known carbodiimide coupling reaction [31]. The first step is the activation of the MUAcarboxyl groups on the surface with a mixture of $0.1 \mathrm{M}$ EDC and $0.05 \mathrm{M}$ NHS to give reactive succinimide esters. BSADNP $(20 \mu \mathrm{g} / \mathrm{mL})$ is then passed over the surface and the esters react spontaneously with primary amine groups of the protein. The immobilization process concludes by blocking remaining succinimide esters using $1 \mathrm{M}$ ethanolamine, $\mathrm{pH}$ 8.5. 


\subsection{Characterization of the Sensor Surfaces}

2.4.1. Thickness Determination by Surface Plasmon Resonance. PLL film thickness was calculated using a two-wavelength method [32]. The experimental SPR curves were fitted with WinSpall 3.02 software based on the Fresnel equations and recursion formalism, freely available from Res-Tec $\mathrm{GmbH}$ [33]. SPR curves of gold substrate were simulated first in order to obtain the effective parameters of the thickness and dielectric constant of bare gold in Milli-Q water before fitting the Au-MUA-PLL curve (Supplementary Information Figure S3) and obtain the layer thickness.

SPR measurements were performed in a BioNavis SPR $\mathrm{Navi}^{\mathrm{TM}} 200$ (MP-SPR). The device is equipped with two independent lasers (670 and $785 \mathrm{~nm}$ ) in both measurement channels on the gold-coated sensing surface and has an angularscan range of 40-78 degrees. The instrument comprises a dual-channel detection system and can make simultaneous measurements in two flow cells.

2.4.2. Atomic Force Microscopy Imaging. Atomic Force Microscopy (AFM) measurements of Au-MUA and AuMUA-PLL were obtained in ambient conditions using a Multimode-Nanoscope V (Veeco, Santa Barbara, CA) operating in Tapping Mode with an etched silicon probe model Arrow-NCR-50 NanoWorld (cantilever resonance frequency: $258 \mathrm{kHz}$, force constant $42 \mathrm{~N} / \mathrm{m}$; tip radius 5-10 nm). Typical scan rates were $1 \mathrm{~Hz}-1.5 \mathrm{~Hz}$.

2.4.3. Surface Enhanced Raman Spectroscopy (SERS) Measurements. Samples of Au-MUA-PLL and Au-MUA-PLLDNP were prepared following the procedure described in Section 2.3 employing nanostructured gold instead of commercial gold substrates in order to enhance Raman signals. The nanostructured metallic substrates consisted in segmented void arrays ( $600 \mathrm{~nm}$ of diameter) and were fabricated following previous reported procedures [34, 35].

SERS measurements were made ex situ using a JobinYvon T64000 triple spectrometer operating in subtractive mode and equipped with a liquid- $\mathrm{N}_{2}$ cooled charge coupled device. The excitation was done with an $\mathrm{Ar}-\mathrm{Kr}$ ion laser at $647.1 \mathrm{~nm}$ wavelength focused into the sample using a microscope objective (20x, 0.4 NA Newport). Typical powers were around $1-2 \mathrm{~mW}$ and $60-120 \mathrm{~s}$ of data acquisition with three accumulations. To check the sample homogeneity, at least three series of measurements were performed in different spots of the sample.

2.5. Nonspecific Binding Monitoring. In order to maximize the detection range of the SPR sensors, the signal from antibody nonspecific binding must be negligible. To evaluate the nonspecific antibody binding to Au-MUA-PLL, the platform was placed in the Surface Plasmon Resonance device and the SPR signal was monitored meanwhile an antibody solution was flowed over the surface.

With respect to the thiol SAMs, as it was mentioned before, in most of the competitive inhibition immunoassays it is necessary to immobilize an analyte-protein derivative to the surface by means of a chemical activation of the carboxylic groups of the thiol and subsequent deactivation of the unreacted-COOH entities. Thus, in these sensor chips, nonspecific antibody binding could occur either to the deactivated carboxylic groups of the thiol SAM or to the carrier protein. In order to evaluate the antibody adsorption to the unreacted carboxylic groups of the thiol self-assembled monolayers, the antibody solution was passed over Au-MUA and Au-MUA/DTT platforms that were previously treated with $0.1 \mathrm{M}$ EDC and $0.05 \mathrm{M}$ NHS and deactivated with ethanolamine $1 \mathrm{M}$.

In all the cases the antibody solutions were $190 \mathrm{nM}$, prepared in PBS + 0.05\% Tween 20 (PBS-T) pH 7.4. The antiDNP solutions were passed during 10 minutes followed by the injection of PBS-T pH 7.4 as running buffer.

Additional SPR experiments were performed in single and mixed thiol SAMs in order to determine the amount of the total antibody binding that corresponds to nonspecific antibody adsorption. With this aim, one of the flow cells of the SPR device served as "sample channel" (Au-MUA-BSA-DNP or Au-MUA/DTT-BSA-DNP) whereas the other worked as a "reference channel" (Au-MUA-BSA or Au-MUA/DTTBSA). Serial dilutions of anti-DNP in PBS-T (ranging from $300 \mathrm{nM}$ to $6 \mu \mathrm{M}$ for Au-MUA and $0.7 \mathrm{nM}$ to $190 \mathrm{nM}$ for Au-MUA/DTT) were injected over the flow cells during ten minutes followed by the injection of PBS-T as running buffer. The measured response on the sample channel was the sum of the specific binding and any nonspecific binding while the response on the reference channel was solely from nonspecific binding.

2.6. Binding Isotherms Curves. SPR binding isotherms curves were performed in SPR fixed angle mode (at the steepest fall angle) using both flow cells simultaneously. All the experiments were carried out at $22^{\circ} \mathrm{C}$ at a flow rate of $10 \mu \mathrm{L} / \mathrm{min}$. Sensorgrams were obtained at different times $(0,6$, and 48 days) using the same Au-MUA-PLL-DNP surface, by passing anti-DNP solutions in PBS-T pH 7.4 at concentrations ranging from $3 \mathrm{nM}$ to $46 \mathrm{nM}$ over the platform, to test the reusability of the sensor chip. Each cycle included a tenminute injection of the antibody solution followed by a 3-minute washing step with PBS-T buffer and, to remove the bound antibody from the sensor surfaces, a 1-minute regeneration step with $100 \mathrm{mM} \mathrm{HCl}$. The SPR signal assigned to each antibody concentration corresponded to the plateau of the SPR response in the time in between the end of the antibody injection and the start of the regeneration step. In our experiments this plateau was reached at $\sim 700 \mathrm{~s}$ of each cycle. Once an experiment was finished, the sensor surface was treated with $\mathrm{HCl} 100 \mathrm{mM}$, washed with Milli-Q water, dried with a stream of nitrogen, and kept at $-20^{\circ} \mathrm{C}$ to perform new experiments with a regenerated surface.

Binding isotherms curves obtained for control experiments with single and mixed thiol SAMs are displayed in Supplementary Information Figures S4 and S5.

2.7. Competitive Inhibition Assays. SPR measurements in the competitive inhibition assays format, where immobilized DNP competes for antibody binding with free DNP in solution, were also carried out. Since the antibody binding 
to the immobilized DNP is inhibited by the presence of the analyte in the solution, increasing concentrations of DNP in solution will reduce the SPR signal. In the present work, the competitive inhibition assays were conducted, as a proof of concept, using $2 \mathrm{mM}$ DNP aqueous solution and antiDNP solutions with concentrations that range from $\mu \mathrm{M}$ to $\mathrm{nM}$, to assure an excess of DNP in the antigen-antibody reaction. Prior to the injection in the SPR device, the mixtures $\mathrm{DNP} /$ anti-DNP were preincubated during 20 minutes at room temperature.

Each cycle included a ten-minute injection of the mixture DNP/anti-DNP followed by a 3-minute washing step with PBS-T pH 7.4 and, to remove the bound antibody from the sensor surfaces, a 1-minute regeneration step with $100 \mathrm{mM} \mathrm{HCl}$.

SPR signal corresponding to each mixture DNP/antiDNP was measured in a time in between the end of the mixture injection and the start of the regeneration step (around 700 s of each cycle).

In order to make a comparison, the same protocol was followed to perform competitive inhibition assays on single and mixed thiol platforms covered with BSA-DNP.

\section{Results and Discussion}

\subsection{Characterization of the Sensor Surfaces}

3.1.1. Thickness Determination by SPR and AFM Imaging. SPR measurements were performed in order to evaluate the thickness of PLL films covalently attached to MUA. A thickness of $30 \pm 1 \AA$ was obtained from the SPR curve fitting, value that is three times higher than the one reported for a PLL monolayer [21]. This difference may be directly related to the average molecular weight of the PLL employed in our work (150-300 kDa) in contrast to the 42 lysine residues-PLL $(9 \mathrm{kDa})$ employed in [21].

AFM images of Au-MUA and Au-MUA-PLL platforms are shown in Figure 1. The topography of both surfaces is similar indicating that the PLL thin film covers the MUA self-assembled monolayer following the nanocrystalline gold surface. Surface roughness measured through the root mean square roughness (RMS) remains practically unchanged for both surfaces $(\mathrm{RMS} \cong 2 \mathrm{~nm}$ for images $1 \times 1 \mu \mathrm{m}$ in size and $3 \mathrm{~nm}$ for images $4 \times 4 \mu \mathrm{m}$ in size).

3.1.2. Raman Spectra. SERS measurements were performed in order to verify the covalent binding of DNP to AuMUA-PLL after 20 hours of incubation with the precursor DNBS. Control spectra were recorded before and immediately after the addition of DNBS to the surface (Figure 2, black and blue curves, resp.). PLL film did not exhibit any spectral features whereas DNBS showed strong signals; in particular bands in $1200-1400 \mathrm{~cm}^{-1}$ region correspond to $-\mathrm{NO}_{2}$ groups [36]. Washing the surface with Milli-Q water after $\sim 1 \mathrm{~h}$ of incubation results in a spectrum that coincides with the one obtained for Au-MUA-PLL surface, evidencing that the measured spectrum (blue curve) corresponded to physisorbed DNBS. A 20-hour incubation period in DNBS solution followed by Milli-Q water washing step achieves the spectrum shown in the red curve of Figure 2. In this case the bands found at 1276, 1313,1339, and $1369 \mathrm{~cm}^{-1}$ are in close resemblance to those found for DNP- $\varepsilon$-lysine, assigned to oand $\mathrm{p}-\mathrm{NO}_{2}$ groups [36] confirming the covalent attachment of the dinitrobenzene group to Au-MUA-PLL.

3.2. Nonspecific Antibody Binding. The sensorgram resulting from the injection of anti-DNP solution over Au-MUA-PLL platform (Figure 3(a)) showed the full recovery of the baseline after the passage of the antibody, verifying the absence of nonspecific antibody binding to the sensor surface. In the case of single and mixed thiol SAMs after the ethanolamine blocking (Figures 3(b) and 3(c)), the SPR signal does not return to the baseline after the passage of the antibody solution, revealing the presence of nonspecific antibody binding to the sensor surfaces.

In order to evaluate if the SPR signal due to the nonspecific antibody adsorption is quantitatively relevant to the SPR change due to the total binding (specific and nonspecific) of anti-DNP to BSA-DNP, the SPR response to the flow of antiDNP solutions over BSA and BSA-DNP covered single or mixed SAMs was assessed. In Figure 4, selected sensorgrams for single (a) and mixed (b) SAMs are presented. Black and red curves give account of nonspecific antibody adsorption and total antibody binding, respectively. The comparison of the SPR signal from the BSA coated thiol surfaces (Figure 4, black curves) and the SPR response corresponding to the uncovered thiol SAMs (Figures 3(b) and 3(c)) verified that the presence of BSA increases the nonspecific antibody binding, probably due to exposure of hydrophobic regions of the carrier protein. Results revealed that, depending on the working anti-DNP concentration, nonspecific adsorption gives account of $25-55 \%$ of the total antibody binding in single SAMs and $48-74 \%$ of the total antibody binding in mixed SAMs.

3.3. Au-MUA-PLL-DNP Binding Isotherm Curves. SPR binding curves for Au-MUA-PLL-DNP platform were obtained by injection of anti-DNP solutions in PBS-T at concentrations ranging from $3 \mathrm{nM}$ to $46 \mathrm{nM}$. In Figure 5 the obtained binding isotherms are shown. Curves corresponding to the freshly prepared, 6-day-old, and 48-day-old Au-MUA-PLLDNP platform are shown in hexagons, stars, and pentagons, respectively. A diminution respect to the freshly made surface, but still persistence of the SPR signal, can be verified after 6 days of the preparation of the platform. After 48 days of the fabrication, almost no SPR response was obtained (pentagons, Figure 5). It should be noted that the Au-MUAPLL-DNP platform was stored at $-20^{\circ} \mathrm{C}$ to be reused in the subsequent experiments. The possibility of reusing Au-MUABSA-DNP or Au-MUA/DTT-BSA-DNP platforms was also attempted resulting in a lack of response even after one day of storage.

3.4. Competitive Inhibition Assays. Competitive inhibition assays were performed on Au-PLL-MUA platforms covered with DNP and, in order to compare, on single and mixed thiol platforms covered with BSA-DNP. The freshly made 


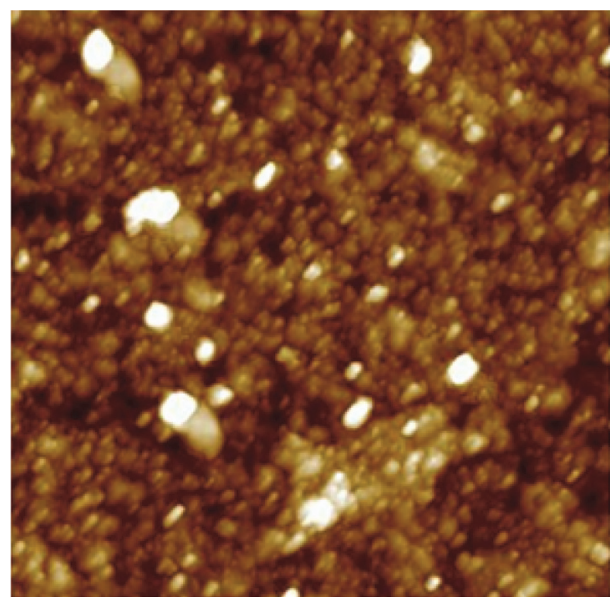

(a)

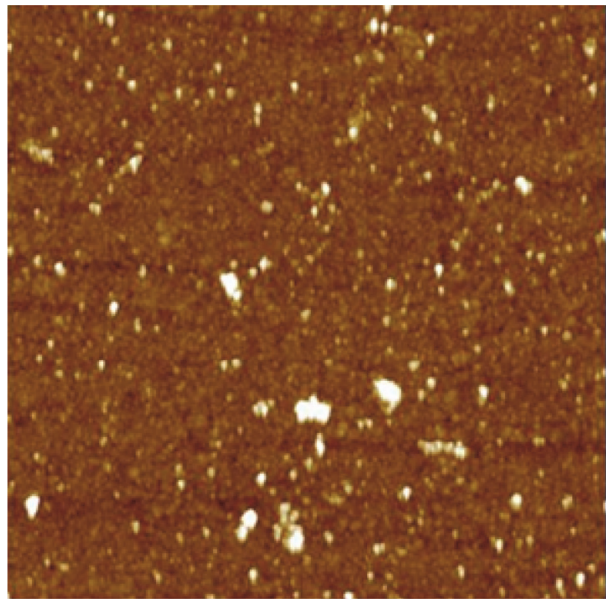

(c)

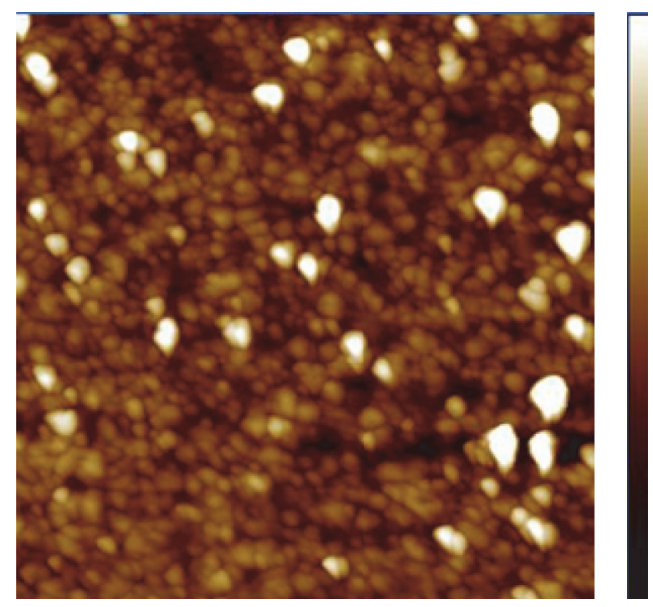

(b)

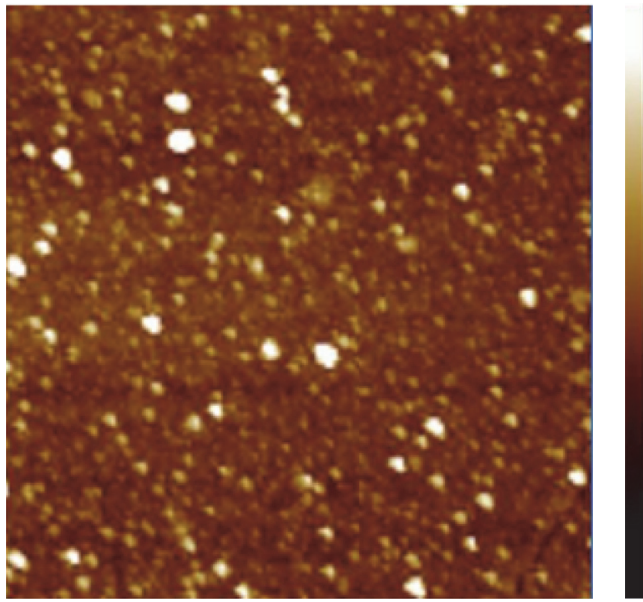

(d)

Figure 1: AFM images of Au-MUA ((a) and (c)) and Au-MUA-PLL ((b) and (d)). Top, $1 \times 1 \mu \mathrm{m}$ and bottom, and $4 \times 4 \mu \mathrm{m}$ in size. Side graduated color bars indicate $z$-scale: white color corresponds to $15 \mathrm{~nm}$ and $40 \mathrm{~nm}$ in top and bottom images, respectively.

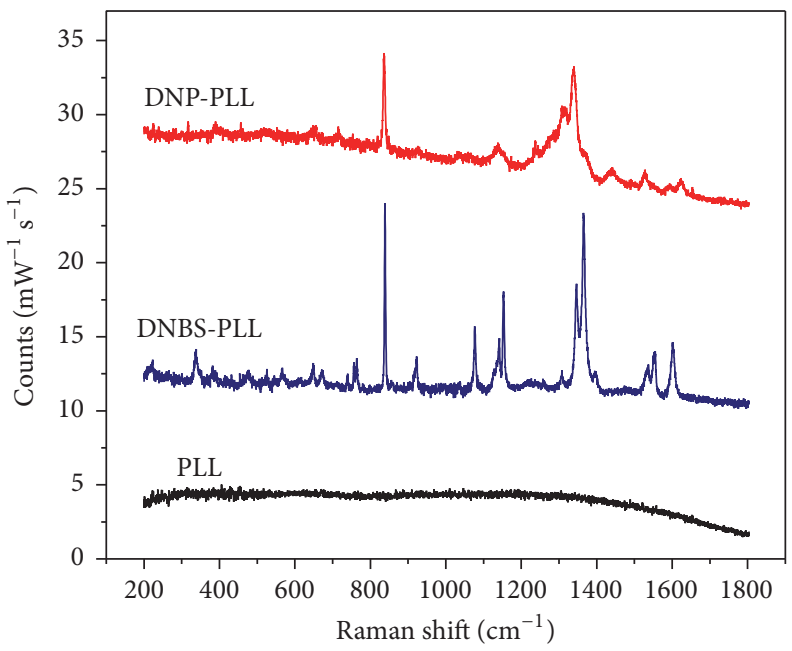

FIGURE 2: SERS spectra of adsorbed DNBS (blue curve) and covalently linked DNP (red curve) on nanostructured gold covered with MUAPLL. Samples were taken in air (dried samples) with the $647.1 \mathrm{~nm}$ laser line. SERS spectrum of Au-MUA-PLL (black curve) was also collected as control experiment. 


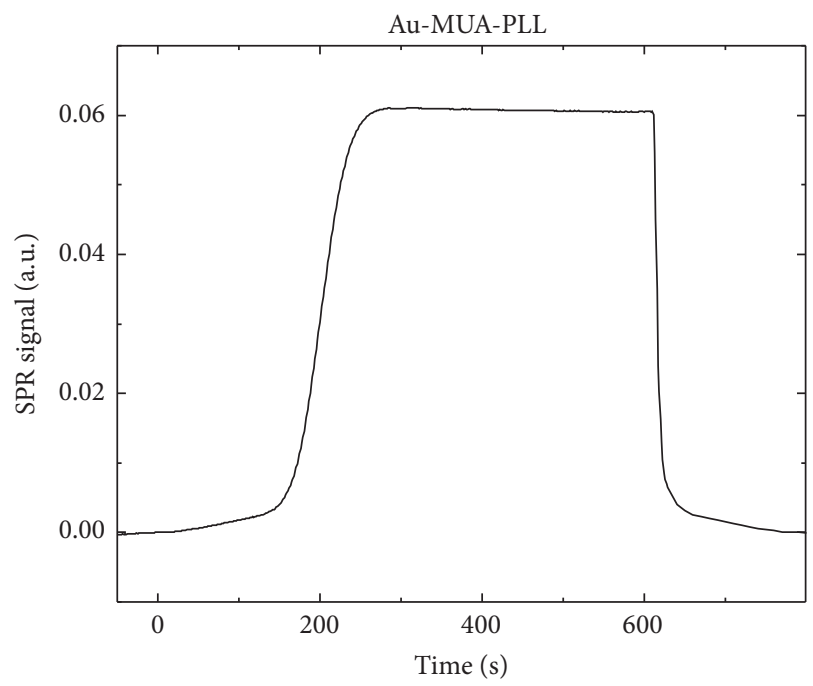

(a)

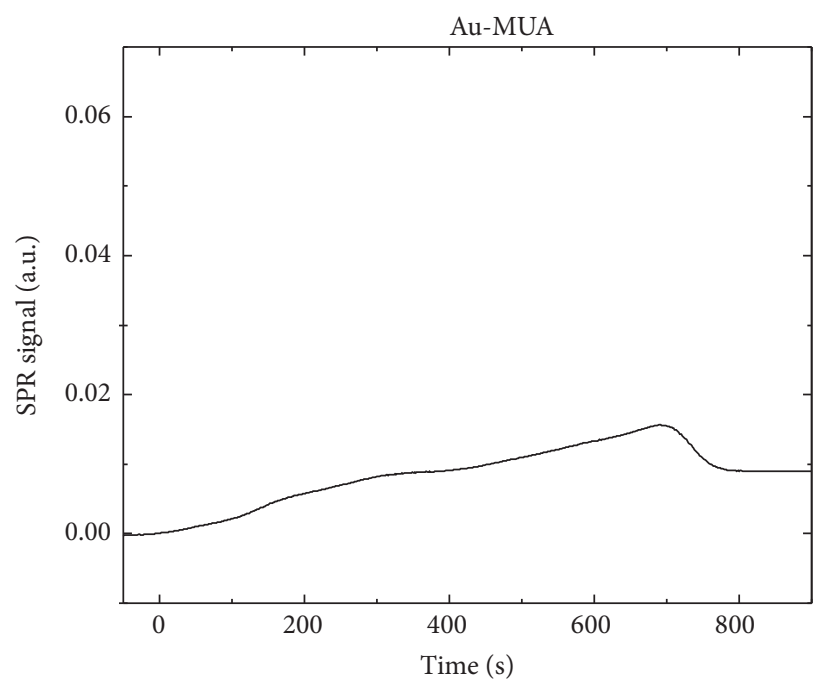

(b)

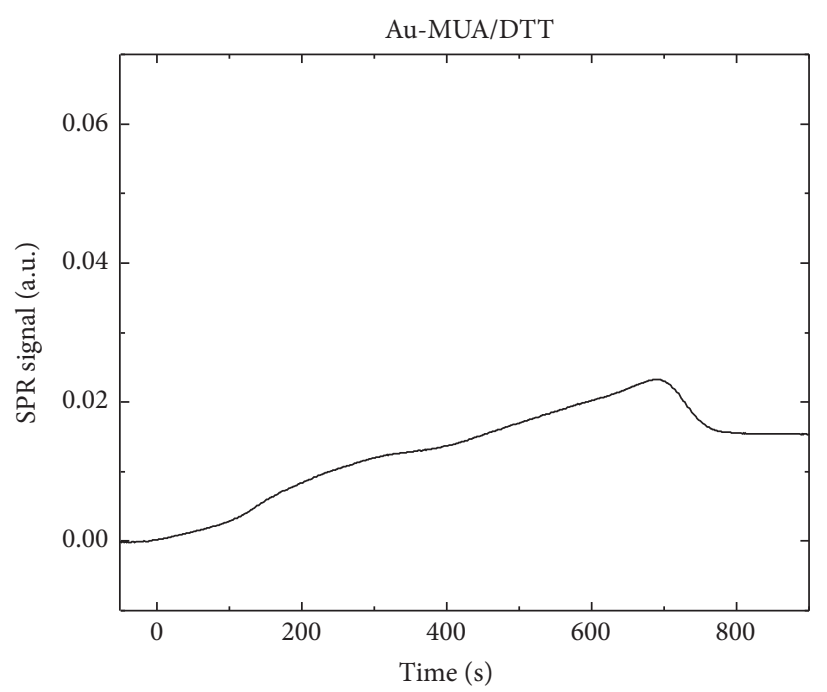

(c)

FIGURE 3: Sensorgrams showing the SPR signal resulting from the injection of anti-DNP solution over (a) Au-MUA-PLL, (b) Au-MUA, and (c) Au-MUA/DTT surfaces. Antibody solution was injected $(t=0)$ during ten minutes followed by running buffer injection. In Au-MUA-PLL, the baseline is fully recovered ( $~ 800 \mathrm{~s})$ after the passage of the antibody verifying the absence of nonspecific antibody binding to the sensor chip. In Au-MUA and Au-MUA/DTT, the SPR signal does not return to the baseline after the injection of the antibody solution, evidencing the presence of nonspecific antibody binding to the surfaces.

Au-MUA-PLL-DNP surface yielded a $67 \%$ inhibition of the maximal SPR signal when $47.5 \mathrm{nM}$ anti-DNP was preincubated with DNP $2 \mathrm{mM}$ during 20 minutes. After six days of preparation, the sensor surface exhibited different degrees of signal inhibition depending on the antibody working concentration (Table 1). Two weeks later, the signal inhibition decreased $10 \%$ with respect to the values reported in Table 1.

Similar competitive inhibition assays in BSA-DNP covered single thiol SAMs resulted in poor degree of signal inhibition (36\% for anti-DNP $38 \mathrm{nM}$ incubated during 20 minutes with DNP $2 \mathrm{mM}$ ) or no inhibition at all in BSADNP immobilized over Au-MUA/DTT. This finding can be related to the high degree of nonspecific antibody binding to the BSA-covered thiol SAMs (reported in Section 3.2.) as the blockage of anti-DNP specific sites by DNP does not prevent the nonspecific adsorption of other regions of antiDNP to the carrier protein or to the unreacted carboxylic groups of the thiol molecules. In this sense, the interactions established within the monoclonal antibody and BSA (or the unreacted-COOH groups) overcome the specific but weak DNP recognition. These interactions could not be prevented even with the use of detergent in the running buffer.

Although poly-L-lysine is also a polypeptide that can have different noncovalent interactions with the antibody molecule, the amount of nonspecific interactions when monoclonal anti-DNP was injected over the platform was negligible. BSA, as most proteins, exhibits a complex surface that can lead to multiple noncovalent protein-protein interactions 


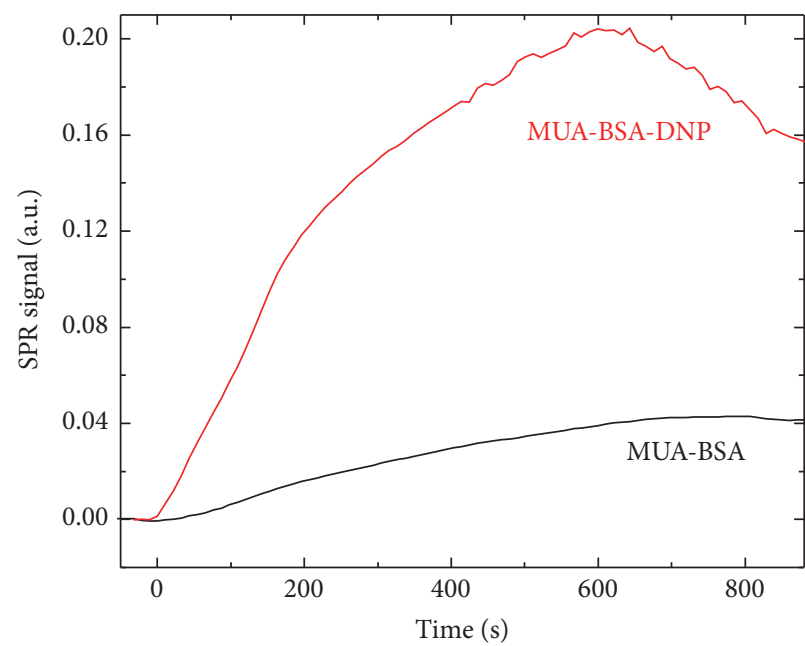

(a)

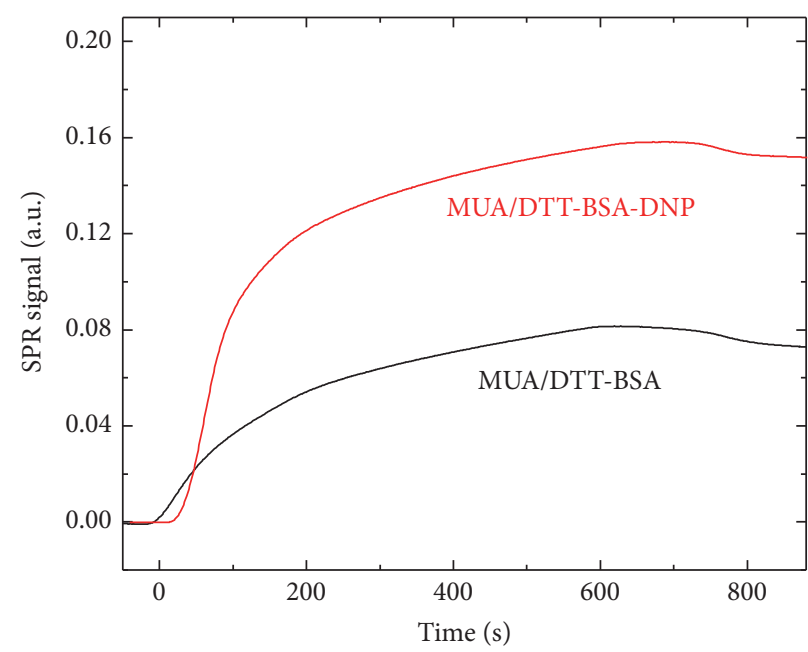

(b)

FIGURE 4: Sensorgrams showing the SPR signal resulting from the injection of anti-DNP solutions over BSA and BSA-DNP coated single and mixed SAMs. In (a) the SPR response of single MUA self-assembled monolayers to the passage of $750 \mathrm{nM}$ anti-DNP is presented. In (b) the SPR change from MUA/DTT SAMs to the flow of $190 \mathrm{nM}$ anti-DNP is displayed. The antibody solutions were injected $(t=0)$ during ten minutes followed by the injection of running buffer. Black and red curves give account of nonspecific antibody adsorption and total antibody binding, respectively. In the selected sensorgrams, nonspecific antibody binding gives account of $26 \%$ of the total antibody binding in single SAM and $48 \%$ of the total antibody binding in mixed SAMs.

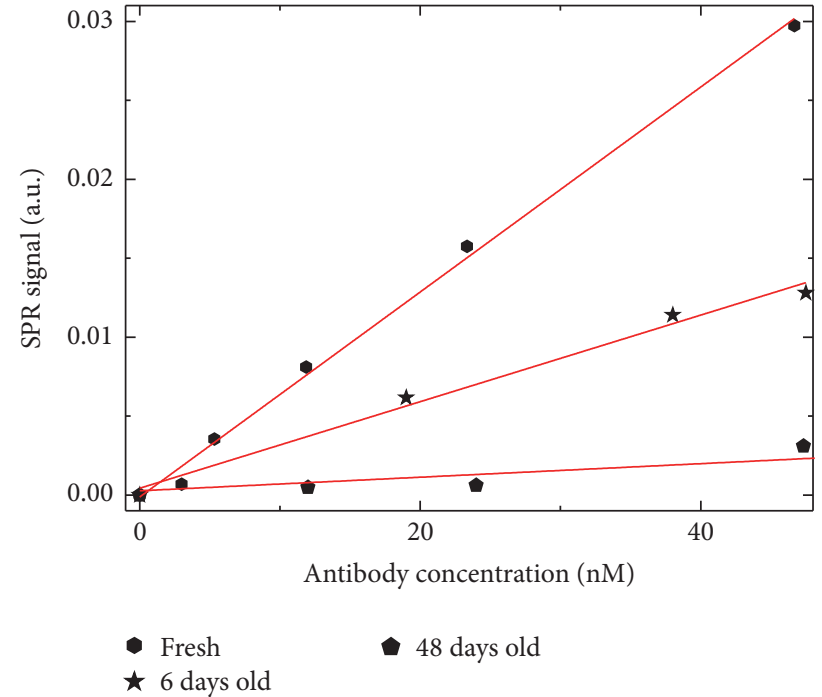

FIGURE 5: SPR binding isotherms obtained by injection of anti-DNP solutions over Au-MUA-PLL-DNP. Hexagons, stars, and pentagons correspond to the freshly made 6-day-old and 48-day-old platform kept at $-20^{\circ} \mathrm{C}$. Each point corresponds to the SPR signal after the end of the antibody injection and before the start of the regeneration step.

[37]. In particular, hydrophobic exposed surfaces that are not present in the poly-L-lysine coated sensor may account for antibody nonspecific binding to BSA coated thiol surfaces, affecting the analytical capacity of the competitive binding assays. These observations should be confirmed using other analyte-antibody pairs; however, it seems reasonable that
TABLE 1: SPR signal inhibition of anti-DNP at different working concentrations when the antibody in solution is preincubated during 20 minutes with DNP $2 \mathrm{mM}$ and the mixture is injected over Au-MUA-PLL-DNP (results correspond to sensor surfaces after 6 days of preparation).

\begin{tabular}{lc}
\hline Antibody concentration & SPR signal inhibition \\
\hline $47.5 \mathrm{nM}$ & $60 \%$ \\
$38 \mathrm{nM}$ & $70 \%$ \\
$19 \mathrm{nM}$ & $82 \%$ \\
\hline
\end{tabular}

the use of a much homogeneous binding surface with no hydrophobic sites exposed, as is the poly-L-lysine coated surface, may represent a good alternative to conjugateprotein attachment to the sensor for competition assays.

\section{Conclusions}

The performance of Au-MUA- poly-L-lysine sensor platforms for quantitative determination of 2,4-dinitrophenol, as model system of small molecule, by Surface Plasmon Resonance measurements in the competitive inhibition test format was addressed. The immobilization of PLL to the sensor chip is achieved in an easier and faster way compared to other reported polymeric coatings that require several days to complete their preparation. PLL films yielded a thickness of $30 \pm 1 \AA$ and, according to AFM imaging, they cover the MUA self-assembled monolayer following the nanocrystalline gold surface. The successful immobilization of 2,4-dinitrophenol to Au-MUA-PLL was verified through SERS measurements. The possibility of directly immobilizing DNP to the poly-L-lysine containing platforms leads to an 
improvement in the detection of the soluble analyte by competitive inhibition assays (when compared with single and mixed thiol SAMs) avoiding undesirable nonspecific protein adsorption. Besides, the possibility of reusing the DNP derivatized platform, after six days of its preparation, by following an adequate regeneration protocol was verified.

Therefore, Au-MUA-PLL surfaces constitute a suitable alternative for quantitative detection of small molecules when nonspecific adsorption cannot be avoided. The advantages of using the Au-MUA-PLL sensor surfaces could be extended to other biorecognition systems where the presence of undesirable protein-protein interactions could interfere.

\section{Competing Interests}

The authors declare that they have no competing interests.

\section{Acknowledgments}

M. Antonieta Daza Millone and Cecilia Y. Chain are members of the research career of CONICET. María E. Vela is member of the research career of CIC PBA. The authors thank Bunge and Born Foundation, CONICET, and INTA for support through grants (PIP 2015, PNNAT-1128043).

\section{References}

[1] R. Karlsson, "SPR for molecular interaction analysis: a review of emerging application areas," Journal of Molecular Recognition, vol. 17, no. 3, pp. 151-161, 2004.

[2] D. R. Shankaran, K. V. Gobi, and N. Miura, "Recent advancements in surface plasmon resonance immunosensors for detection of small molecules of biomedical, food and environmental interest," Sensors and Actuators, B: Chemical, vol. 121, no. 1, pp. 158-177, 2007.

[3] J. Homola, "Surface plasmon resonance sensors for detection of chemical and biological species," Chemical Reviews, vol. 108, no. 2, pp. 462-493, 2008.

[4] N. J. de Mol and M. J. E. Fischer, "Kinetic and thermodynamic analysis of ligand-receptor interactions: SPR applications in drug development," in Handbook of Surface Plasmon Resonance, chapter 5, pp. 123-172, The Royal Society of Chemistry, 2008.

[5] Y. Uludag and I. E. Tothill, "Cancer biomarker detection in serum samples using surface plasmon resonance and quartz crystal microbalance sensors with nanoparticle signal amplification," Analytical Chemistry, vol. 84, no. 14, pp. 5898-5904, 2012.

[6] T. Onodera and K. Toko, "Towards an electronic dog nose: surface plasmon resonance immunosensor for security and safety," Sensors (Basel, Switzerland), vol. 14, no. 9, pp. 16586-16616, 2014.

[7] D. R. Shankaran, K. Matsumoto, K. Toko, and N. Miura, "Development and comparison of two immunoassays for the detection of 2,4,6-trinitrotoluene (TNT) based on surface plasmon resonance," Sensors and Actuators, B: Chemical, vol. 114, no. 1, pp. 71-79, 2006.

[8] J. Homola, "Surface plasmon resonance based sensors," in Methods and Applications, O. S. Wolfbei, Ed., Springer Series on Chemical Sensors and Biosensors, Springer, Berlin, Germany, 2006.
[9] J. Mitchell, "Small molecule immunosensing using surface plasmon resonance," Sensors, vol. 10, no. 8, pp. 7323-7346, 2010.

[10] N. J. de Mol and M. J. Fischer, Surface Plasmon Resonance Methods and Protocols, Springer, London, UK, 2010.

[11] X. Guo, "Surface plasmon resonance based biosensor technique: a review," Journal of Biophotonics, vol. 5, no. 7, pp. 483-501, 2012.

[12] H. H. Nguyen, J. Park, S. Kang, and M. Kim, "Surface plasmon resonance: a versatile technique for biosensor applications," Sensors (Switzerland), vol. 15, no. 5, pp. 10481-10510, 2015.

[13] N. Miura, K. Ogata, G. Sakai, T. Uda, and N. Yamazoe, "Detection of morphine in ppb range by using SPR (SurfacePlasmon-Resonance) immunosensor," Chemistry Letters, vol. 26, no. 8, pp. 713-714, 1997.

[14] K. V. Gobi, H. Tanaka, Y. Shoyama, and N. Miura, "Continuous flow immunosensor for highly selective and real-time detection of sub-ppb levels of 2-hydroxybiphenyl by using surface plasmon resonance imaging," Biosensors and Bioelectronics, vol. 20, no. 2, pp. 350-357, 2004.

[15] Q. Yu, S. Chen, A. D. Taylor, J. Homola, B. Hock, and S. Jiang, "Detection of low-molecular-weight domoic acid using surface plasmon resonance sensor," Sensors and Actuators, B: Chemical, vol. 107, no. 1, pp. 193-201, 2005.

[16] S. J. Daly, G. J. Keating, P. P. Dillon et al., "Development of surface plasmon resonance-based immunoassay for aflatoxin B1," Journal of Agricultural and Food Chemistry, vol. 48, no. 11, pp. 5097-5104, 2000.

[17] S. J. Kim, K. V. Gobi, R. Harada, D. R. Shankaran, and N. Miura, "Miniaturized portable surface plasmon resonance immunosensor applicable for on-site detection of low-molecular-weight analytes," Sensors and Actuators B: Chemical, vol. 115, no. 1, pp. 349-356, 2006.

[18] M.-C. Estevez, J. Belenguer, S. Gomez-Montes et al., "Indirect competitive immunoassay for the detection of fungicide Thiabendazole in whole orange samples by Surface Plasmon Resonance," Analyst, vol. 137, no. 23, pp. 5659-5665, 2012.

[19] J.-F. Masson, T. M. Battaglia, Y.-C. Kim, A. Prakash, S. Beaudoin, and K. S. Booksh, "Preparation of analyte-sensitive polymeric supports for biochemical sensors," Talanta, vol. 64, no. 3, pp. 716-725, 2004.

[20] J.-F. Masson, T. M. Battaglia, M. J. Davidson et al., "Biocompatible polymers for antibody support on gold surfaces," Talanta, vol. 67, no. 5, pp. 918-925, 2005.

[21] B. L. Frey and R. M. Corn, "Covalent attachment and derivatization of poly(l-lysine) monolayers on gold surfaces as characterized by polarization-modulation FT-IR spectroscopy," Analytical Chemistry, vol. 68, pp. 3187-3193, 1996.

[22] M. Park, J.-H. Bong, G. Yoo, J. Jose, M.-J. Kang, and J.-C. Pyun, "Optimization of a FACS based-immunoassay using $E$. coli autodisplaying Z-domains," Biochip Journal, vol. 7, no. 2, pp. 173-179, 2013.

[23] H. Aizawa, M. Tozuka, S. Kurosawa, K. Kobayashi, S. M. Reddy, and M. Higuchi, "Surface plasmon resonance-based trace detection of small molecules by competitive and signal enhancement immunoreaction," Analytica Chimica Acta, vol. 591, no. 2, pp. 191-194, 2007.

[24] P. Singh, T. Onodera, Y. Mizuta, K. Matsumoto, N. Miura, and K. Toko, "Novel DNP-KLH protein conjugate surface for sensitive detection of TNT on SPR immunosensor," Sensors and Materials, vol. 19, no. 5, pp. 261-273, 2007.

[25] T. M. Chinowsky, S. D. Soelberg, P. Baker et al., "Portable 24-analyte surface plasmon resonance instruments for rapid, 
versatile biodetection," Biosensors and Bioelectronics, vol. 22, pp. 2268-2275, 2007.

[26] T. Yang, P. Zhong, L. Qu, C. Wang, and Y. Yuan, "Preparation and identification of anti-2, 4-dinitrophenyl monoclonal antibodies," Journal of Immunological Methods, vol. 313, no. 1-2, pp. 20-28, 2006.

[27] L. Morelli, L. Plotkin, J. Leoni, C. A. Fossati, and R. A. Margni, "Analysis of oligosaccharides involved in the asymmetrical glycosylation of igg monoclonal antibodies," Molecular Immunology, vol. 30, no. 7, pp. 695-700, 1993.

[28] G. Galfrè and C. Milstein, "Preparation of monoclonal antibodies: strategies and procedures," Methods in Enzymology, vol. 73, pp. 3-46, 1981.

[29] H. N. Eisen, L. Orris, and S. Belman, "Elicitation of delayed allergic skin reactions with haptens: the dependence of elicitation on hapten combination with protein," Journal of Experimental Medicine, vol. 95, no. 5, pp. 473-487, 1952.

[30] C. W. Parker, M. Kern, and H. N. Eisen, "Polyfunctional dinitrophenyl haptens as reagents for elicitation of immediate type allergic skin responses," The Journal of Experimental Medicine, vol. 115, no. 4, p. 789, 1962.

[31] Z. Grabarek and J. Gergely, "Zero-length crosslinking procedure with the use of active esters," Analytical Biochemistry, vol. 185, no. 1, pp. 131-135, 1990.

[32] H. Liang, H. Miranto, N. Granqvist et al., "Surface plasmon resonance instrument as a refractometer for liquids and ultrathin films," Sensors and Actuators B: Chemical, vol. 149, no. 1, pp. 212220, 2010.

[33] http://www.res-tec.de/downloads.html.

[34] P. N. Bartlett, J. J. Baumberg, S. Coyle, and M. E. Abdelsalam, "Optical properties of nanostructured metal films," Faraday Discussions, vol. 125, pp. 117-132, 2004.

[35] L. A. Guerra Hernández, M. A. Daza Millone, E. Cortés et al., "Synergetic light-harvesting and near-field enhancement in multiscale patterned gold substrates," ACS Photonics, vol. 2, no. 9, pp. 1355-1365, 2015.

[36] K. Kumar, D. J. Phelps, P. R. Carey, and N. M. Young, "Resonance raman-spectroscopic studies of the hapten features involved in the binding of 2,4-dinitrophenyl haptens by the mouse myeloma proteins MOPC 315 and MOPC 460," Biochemical Journal, vol. 175, no. 2, pp. 727-735, 1978.

[37] J. De Las Rivas and C. Fontanillo, "Protein-protein interactions essentials: key concepts to building and analyzing interactome networks," PLoS Computational Biology, vol. 6, no. 6, Article ID e1000807, 2010. 

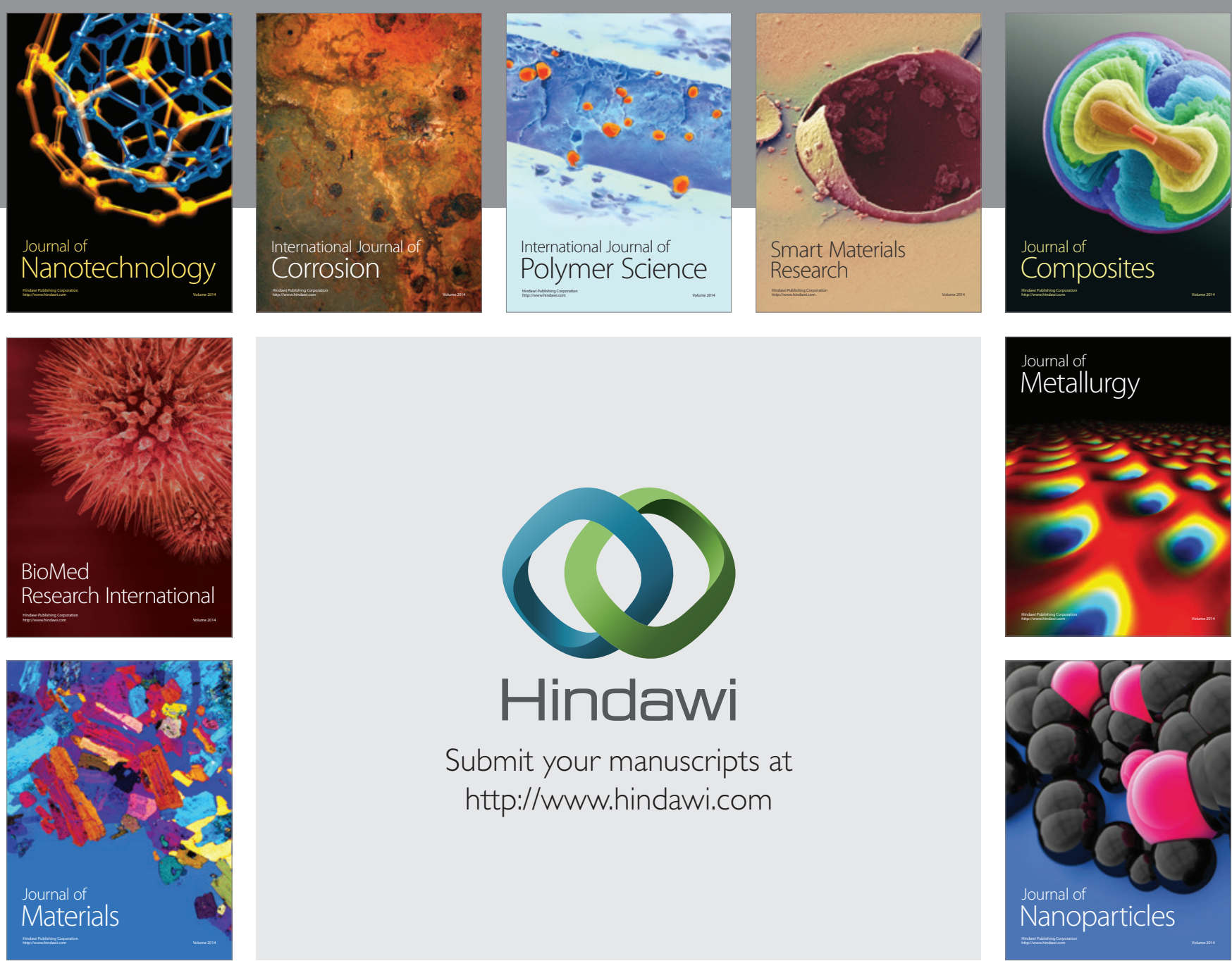

\section{Hindawi}

Submit your manuscripts at

http://www.hindawi.com

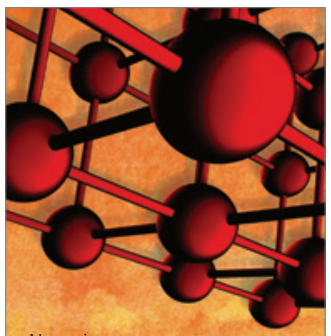

Materials Science and Engineering
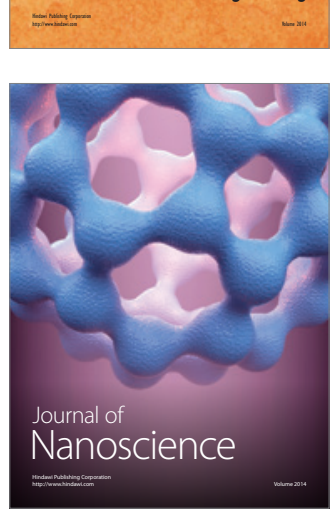
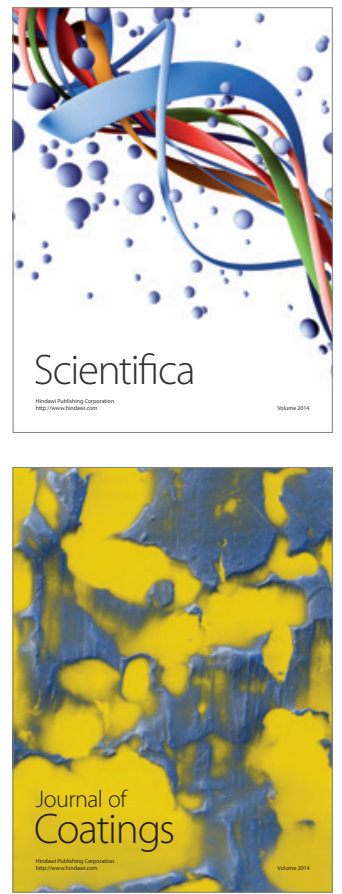
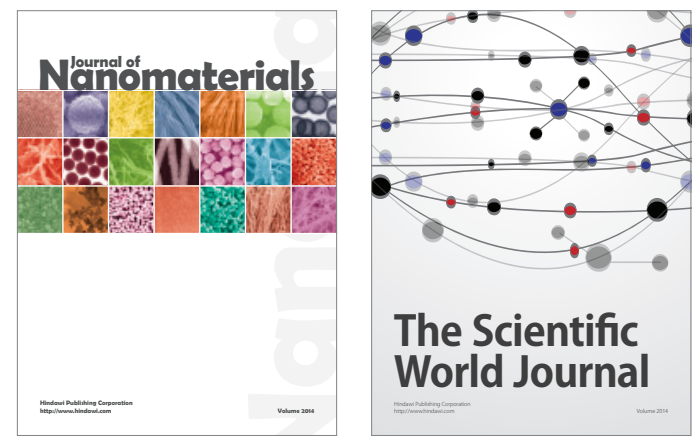

The Scientific World Journal
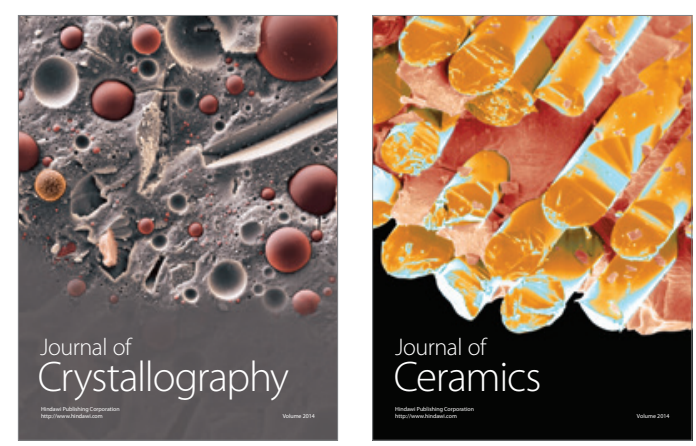
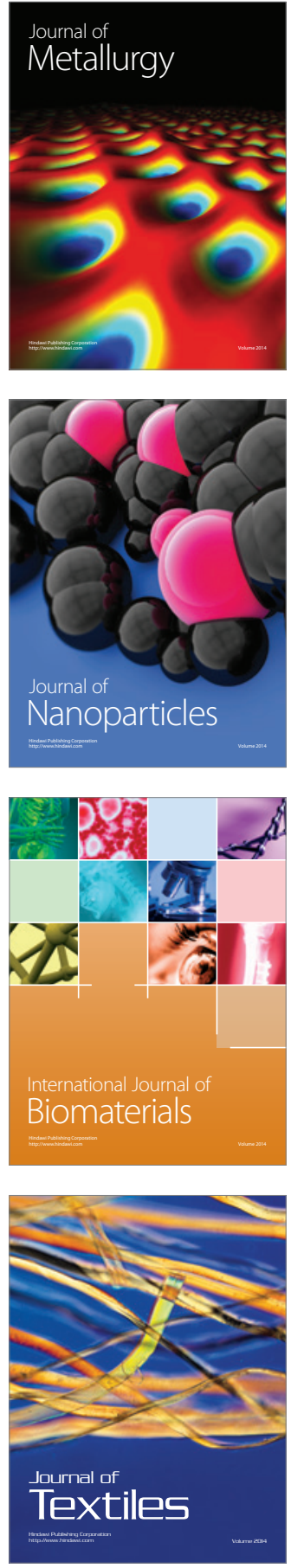